\title{
THRESHOLD RESONANCE IN GEOMETRIC SCATTERING
}

\author{
Xue Ping Wang *
}

\begin{abstract}
We study the properties of the resonant states at 0 for Schrödinger operators of the form $P=-\Delta_{g}+\frac{q(\theta)}{r^{2}}+V_{0}(x)$ on a Riemannian manifold, where $x=r \theta$ is some polar coordinates and $g$ is a perturbation of a Riemannian metric $g_{0}=d r^{2}+r^{2} h$ with $h$ independent of $r$. A characterization of zero energy resonant states is given in terms of small eigenvalues of $-\Delta_{h}+q(\theta)$.
\end{abstract}

\section{Introduction}

The spectral analysis of two-body Schrödinger-type operators at the threshold zero has a long history and has been studied in many works (see for example $[1,4,14,15,19]$ and the references therein). These works are concerned with perturbation of a constant elliptic differential operator (in most cases, the perturbation of Laplacian on $\mathbb{R}^{n}$ ) by a term decaying like $O\left(|x|^{-2-\epsilon}\right), \epsilon>0$, as $x \rightarrow \infty$. The decay condition is needed to introduce the notion of threshold energy resonance. The spectral analysis of $N$-body Schrödinger operators at its first threshold, the bottom of the essential spectrum, is studied in [23] where some effective potential decays with the same rate in intercluster variables. The relevant issue in these works is the asymptotic expansions of resolvent near the threshold. The main difficulty arises from the possible existence of zero energy

*The author thanks G. Carron for helpful discussions and the organizers of Symposium "Scattering and Spectral theory" in Serrembi, Brazil, for their kind invitation. Research supported in part by a grant of Outstanding Overseas Chinese Scholars of Chinese Academy of Sciences.

2000 Mathematics Subject Classification. 35P25, 47A40, 81U10

Key words and phrases. threshold energy resonance, geometric scattering, Schrödinger operators on manifold 
resonant states, which are invisible in the $L^{2}$-setting. Thresholds are exceptional points where new physical phenomena appear. The understanding of resonant states at thresholds plays an important role in the study of complex phenomena $([1,2,19,24])$. In this paper, we shall analyze the zero energy resonant states in a geometric scattering. The result of this paper is to be used in [25] to establish the low-energy asymptotic expansion of the resolvent, and under an appropriate non-trapping condition, the long-time expansion of wave functions for the time-dependent Schrödinger equation.

The analysis of zero energy resonant states is also of interest in Riemannian geometry. In fact, the extended index of a Dirac-type operator, $D$, on a non compact Riemannian manifold, $M$, can be written as sum of the local contribution from Atiyah-Singer index theorem and the contribution from the infinity $[3,11,17]$. If $D$ is non parabolic at the infinity, and if $E \rightarrow M$ is a $\mathbb{Z}_{2}$-graded vector bundle, $E=E^{+} \oplus E^{-}$, then there exists some Hilbert space $W\left(E^{+}\right) \subset H_{l o c}^{1}\left(E^{+}\right)$such that $D^{+}: W\left(E^{+}\right) \rightarrow L^{2}\left(E^{-}\right)$is Fredholm and the extended index can be then defined as:

$$
\operatorname{ind}_{e} D^{+}=\operatorname{dim}\left(\operatorname{ker}_{W} D^{+} / \operatorname{ker}_{L^{2}} D^{+}\right)+\operatorname{ind}_{L^{2}} D^{+} .
$$

The elements $u \in \operatorname{ker}_{W} D^{+} / \operatorname{ker}_{L^{2}} D^{+}$can be identified as co-closed zero energy resonant states of some Laplace-type operator.

Our work on this subject is motivated by the paper of G. Carron [11] and is concerned with the low-energy spectral analysis for perturbation of a class of metrics. We want to study Schrödinger operator $P=-\Delta_{g}+W(x)$ which is perturbation of an operator $P_{0}$ of the form

$$
P_{0}=-\Delta_{g_{0}}+\frac{q(\theta)}{r^{2}}
$$

on a Riemannian manifold $M$, with dimension $n \geq 2$, which is, outside some compact, isometric to a conical space $\mathbb{R}_{+} \times \Sigma, \Sigma$ being a compact $(n-1)$ dimensional manifold with or without boundary. If $\boldsymbol{\Sigma}$ is of boundary, the Dirich- 
let condition is used. Here $(r, \theta) \in \mathbb{R}_{+} \times \boldsymbol{\Sigma}$ is the polar coordinates, $q(\theta)$ is a real continuous function and $g_{0}$ is a metric on $\mathbb{R}_{+} \times \boldsymbol{\Sigma}$ of the form

$$
g_{0}=d r^{2}+r^{2} h(\theta, d \theta)
$$

with $h$ an arbitrary Riemannian metric on $\boldsymbol{\Sigma}$ independent of $r$. The term $\frac{q(\theta)}{r^{2}}$ can not be treated by method of perturbation previously used in low-energy spectral analysis. New phenomenon occurs due to the non trivial metric $h$. Assume $-\Delta_{h}+q(\theta) \geq-\frac{(n-2)^{2}}{4}$. Set

$\sigma_{\infty}=\left\{\nu ; \nu=\sqrt{\lambda+\frac{(n-2)^{2}}{4}}, \lambda \in \sigma\left(-\Delta_{h}+q\right)\right\}, \quad \sigma_{k}=\sigma_{\infty} \cap[0, k], \quad k \in \mathbb{N}$.

When $h=(d \theta)^{2}$, the standard Euclidean metric on the sphere $\mathbb{S}^{n-1}$ with $n \geq 2$ and $q=0$, one has $P_{0}=-\Delta$ and

$$
\sigma_{\infty}=\left\{\frac{n-2}{2}+k ; k \in \mathbb{N}\right\} .
$$

In this case, $\sigma_{\infty}$ consists of either only half-integers ( $n$ odd) or only integers ( $n$ even). In particular, for Laplace operator $-\Delta$ on $\mathbb{R}^{n}$, one has

$$
\sigma_{1}=\left\{\begin{array}{cc}
\{0,1\}, & n=2 \\
\left\{\frac{1}{2}\right\}, & n=3, \\
\{1\}, & n=4 \\
\emptyset, & n \geq 5
\end{array}\right.
$$

For perturbation of Euclidean Laplacian $-\Delta$, it is well-known (see, for example, $[4,15])$ that there are at most one $s$-wave resonant state and two $p$-wave resonant states for $n=2$, and one $s$-wave resonant state for $n=3,4$ and no zero energy resonant state for $n \geq 5$. In particular, there is no $d$-wave resonant state for the perturbation of the Euclidean Laplacian for all $n \geq 2$. In terms of the results of this paper, these properties can be regarded as a consequence of special spectral properties (1.4) of the Laplacian with the standard metric on $\mathbb{S}^{n-1}$. In the perturbation of a non-trivial metric $h$, the multiplicity of zero resonance can be arbitrarily large. Even in the simple case where $h$ is of the form

$$
h=\hbar^{-2}(d \theta)^{2}, \quad \hbar>0
$$


$(d \theta)^{2}$ being the Euclidean metric on the sphere, there may be resonant states with any large wave numbers as $\hbar \rightarrow 0$. In Section 5 , we shall show that the multiplicity of the zero resonance has a lower bound of the form $C \hbar^{-(n-1)}, C>$ 0 , for the Hodge-de Rham Laplacian associated with a family of $\hbar$-dependent metrics on $\Sigma$.

In [11], G. Carron studied the operator $P$, with $P=P_{0}$ outside some compact $K$ in a Riemannian manifold $(M, g)$ with conical end. He obtained an interesting formula on the jump at 0 for the spectral shift function $\xi\left(\lambda ; P, P_{M \backslash K}\right)$ :

$$
\xi\left(0_{+} ; P, P_{M \backslash K}\right)-\xi\left(0_{-} ; P, P_{M \backslash K}\right)=\sum_{\nu \in \sigma_{1}} \nu m_{\nu}+\operatorname{dim}_{\operatorname{ker}_{L^{2}}} P
$$

where $m_{\nu}$ is the dimension of resonant states of $P$ with energy 0 behaving like

$$
\frac{\varphi(\theta)}{r^{\frac{n-2}{2}+\nu}}(1+o(1)), \quad r \rightarrow \infty, \quad \text { for some } \varphi \neq 0, \nu \in \sigma_{1} \text {. }
$$

(1.5) is proved in [11] by the method of Dirichlet-to-Neumann operator and shows that each resonant state of the behavior (1.6) gives a contribution $\nu$ to the jump of the spectral shift function at 0 . This is to compare with the wellknown phenomenon for Schrödinger operators on $\mathbb{R}^{3}$ where the resonant state at 0 gives a contribution of $1 / 2$ to this jump. The goal of this work is to analyze the zero energy resonant states in more general situation. The main difference from the previous works $[1,4,14,19]$ on perturbation of constant elliptic operators is the presence of a non-trivial metric $h$.

The plan of this work is as follows. In Section 2, we give the asymptotic expansions of the resolvent $R_{0}(z)=\left(P_{0}-z\right)^{-1}$. As a consequence, we obtain in Section 3 the asymptotic expansion of wave functions associated to $P_{0}$. Note that the study of the model operator $P_{0}$ is already non trivial, although the kernel of the resolvent $R_{0}(z)$ can be expressed in terms of Bessel functions. Section 4 is devoted to the characterization for resonant states at zero for $P$. We show that the behavior of resonant states with zero energy is governed by small eigenvalues of $-\Delta_{h}+q(\theta)$. This result is important to the proof of the existence of asymptotic expansions of the resolvent $(P-z)^{-1}$ at $0([25])$. We 
give in Section 5 a semi-classical estimate for the multiplicity of the zero resonance. Finally, asymptotic expansions for some oscillatory integrals needed in this work are given in an appendix.

Notation. The scalar product on $L^{2}\left(\mathbb{R}_{+} ; r^{n-1} d r\right)$ and $L^{2}\left(M ; d v_{g}\right)$ is denoted by $<\cdot, \cdot>$ and that on $L^{2}\left(\boldsymbol{\Sigma} ; d v_{h}\right)$ by $(\cdot, \cdot) . H^{r, s}(M), r \in \mathbb{Z}, s \in \mathbb{R}$, denotes the weighted Sobolev space of order $r$ with volume element $\langle x\rangle^{2 s} d v \cdot H_{0}^{1, s}$ denote the subspace of $H^{1, s}$ with trace zero on the boundary. The duality between $H^{1, s}$ and $H^{-1,-s}$ is identified with $L^{2}$-product. Denote $H^{0, s}=L^{2, s} \cdot \mathcal{L}\left(r, s ; r^{\prime}, s^{\prime}\right)$ stands for the space of continuous linear operators from $H^{r, s}$ to $H^{r^{\prime}, s^{\prime}}$. In this work, $P$ and $P_{0}$ are considered as self-adjoint operators in $L^{2}$ with form domain $H^{1}$. The complex plane $\mathbb{C}$ is slit along positive real axis so that $z^{\nu}=e^{\nu \ln z}$ and $\ln z=\log |z|+i \arg z$ with $0<\arg z<2 \pi$ are holomorphic there.

\section{Resolvent of the unperturbed operator}

Consider the operator

$$
P_{0}=-\Delta_{g_{0}}+\frac{q(\theta)}{r^{2}}
$$

on $M_{0}=\mathbb{R}_{+} \times \boldsymbol{\Sigma}$, where $\boldsymbol{\Sigma}$ is an $(n-1)$-dimensional compact manifold, $n \geq 2$. Here $(r, \theta) \in \mathbb{R}_{+} \times \boldsymbol{\Sigma}, q(\theta)$ is a real continuous function and $g_{0}$ a metric of the form

$$
g_{0}=d r^{2}+r^{2} h
$$

with $h$ a Riemannian metric on $\boldsymbol{\Sigma}$ independent of $r$. If $\boldsymbol{\Sigma}$ is of boundary, the Dirichlet condition is used for $P_{0}$. Let $\Delta_{h}$ denote Laplace-Beltrami operator on $\Sigma$. Assume

$$
-\Delta_{h}+q(\theta) \geq-\frac{(n-2)^{2}}{4}, \quad \text { on } L^{2}(\boldsymbol{\Sigma}) .
$$

Then, $P_{0} \geq 0$ in $L^{2}\left(M_{0} ; d v\right)$ (see [11]). One has

$$
P_{0}=-\frac{\partial^{2}}{\partial r^{2}}-\frac{n-1}{r} \frac{\partial}{\partial r}+\frac{1}{r^{2}}\left(-\Delta_{h}+q(\theta)\right)
$$


Put

$$
\sigma_{\infty}=\left\{\nu ; \nu=\sqrt{\lambda+\frac{(n-2)^{2}}{4}}, \lambda \in \sigma\left(-\Delta_{h}+q\right)\right\} .
$$

Denote

$$
\sigma_{k}=\sigma_{\infty} \cap[0, k], \quad k \in \mathbb{N} .
$$

For $\nu \in \sigma_{\infty}$, let $n_{\nu}$ denote the multiplicity of $\lambda_{\nu}=\nu^{2}-\frac{(n-2)^{2}}{4}$ as eigenvalue of $-\Delta_{h}+q(\theta)$. Let $\left\{\varphi_{\nu}^{(j)} ; \nu \in \sigma_{h, q}, 1 \leq j \leq n_{\nu}\right\}$ denote an orthonormal basis of $L^{2}(\boldsymbol{\Sigma})$ consisting of eigenfunctions of $-\Delta_{h}+q(\theta)$ :

$$
\left(-\Delta_{h}+q(\theta)\right) \varphi_{\nu}^{(j)}=\lambda_{\nu} \varphi_{\nu}^{(j)}, \quad\left(\varphi_{\nu}^{(i)}, \varphi_{\nu}^{(j)}\right)=\delta_{i j} .
$$

Let $\pi_{\nu}$ denote the orthogonal projection in $L^{2}\left(M_{0}\right)$ onto the subspace spanned by the eigenfunction of $-\Delta_{h}+q$ associated with the eigenvalue $\lambda_{\nu}$

$$
\pi_{\nu} f=\sum_{j=1}^{n_{\nu}}\left(f, \varphi_{\nu}^{(j)}\right) \otimes \varphi_{\nu}^{(j)}, \quad f \in L^{2}\left(M_{0}\right)
$$

Set

$$
Q_{\nu}=-\frac{d^{2}}{d r^{2}}-\frac{n-1}{r} \frac{d}{d r}+\frac{\nu^{2}-\frac{(n-2)^{2}}{4}}{r^{2}}, \quad \text { in } L^{2}\left(\mathbb{R}_{+} ; r^{n-1} d r\right) .
$$

Then, we have the orthogonal decomposition for the resolvent $R_{0}(z)=\left(P_{0}-z\right)^{-1}$

$$
R_{0}(z)=\sum_{\nu \in \sigma_{\infty}}\left(Q_{\nu}-z\right)^{-1} \pi_{\nu}, \quad z \notin \mathbb{R} .
$$

To establish the asymptotic expansion of the resolvent $R_{0}(z)$ for $z$ near 0 , we look for the asymptotic expansion as $z \rightarrow 0$ for each $\left(Q_{\nu}-z\right)^{-1}$ and estimate the remainders w.r.t. $\nu$. Remark that $Q_{\nu}$ can be diagonalized by the Hankel transform of order $\nu$ ([26]). The Schwartz kernel of $\left(Q_{\nu}-z\right)^{-1}$ is given by:

$$
\begin{aligned}
K_{\nu}(r, \tau ; z) & =-(r \tau)^{-\frac{n-2}{2}} \int_{0}^{\infty} e^{-\frac{r^{2}+\tau^{2}}{4 i t}+i z t-i \frac{\pi \nu}{2}} J_{\nu}\left(\frac{r \tau}{2 t}\right) \frac{d t}{2 t} \\
& =-(r \tau)^{-\frac{n-2}{2}} \int_{0}^{\infty} e^{i \frac{\rho}{t}+i z r \tau t-i \frac{\pi \nu}{2}} J_{\nu}\left(\frac{1}{2 t}\right) \frac{d t}{2 t}
\end{aligned}
$$

for $(r, \tau) \in \mathbb{R}_{+}^{2}$ and $z$ with $\Im z>0$ (see [11] and [21]). Here $J_{\nu}$ is the Bessel function of the first kind of order $\nu$ and

$$
\rho=\rho(r, \tau) \equiv \frac{r^{2}+\tau^{2}}{4 r \tau}
$$


$J_{\nu}$ can be represented as

$$
J_{\nu}(\lambda)=\frac{1}{\sqrt{\pi} \Gamma(\nu+1 / 2)}\left(\frac{\lambda}{2}\right)^{\nu} \int_{-1}^{1} e^{i \lambda t}\left(1-t^{2}\right)^{\nu-1 / 2} d t, \quad \Re \nu>-1 / 2,
$$

and has the asymptotic behavior: $J_{\nu}(\lambda)=O\left(\lambda^{\nu}\right)$ as $\lambda \rightarrow 0, J_{\nu}(\lambda)=O\left(\lambda^{-1 / 2}\right)$ as $\lambda \rightarrow \infty$. See $[20,26]$.

We first give formal expansion for resolvent $\left(Q_{\nu}-z\right)^{-1}, \nu \in \sigma_{\infty}$, in the sense of pointwise convergence of their Schwartz kernels. By an abuse of notation, we use the same letter $K$ to denote the operator defined by the Schwartz kernel $K(r, \tau)$ in $L^{2}\left(\mathbb{R}_{+}, r^{n-1} d r\right)$. Let $\nu \in \sigma_{\infty}$ and $l \in \mathbb{N}$ with $l \leq \nu<l+1$. If $l \geq 1$, $K_{\nu}(z)$ can be first expanded in the form

$$
\begin{aligned}
K_{\nu}(r, \tau ; z) & =\sum_{k=0}^{l-1} z^{k} F_{\nu, k}(r, \tau)+R_{\nu, l-1}(r, \tau ; z) \\
F_{\nu, k}(r, \tau) & =-(r \tau)^{-\frac{n-2}{2}+k} \frac{i^{k}}{k !} \int_{0}^{\infty} e^{i \frac{\rho}{t}-i \frac{\pi \nu}{2}} t^{k} J_{\nu}\left(\frac{1}{2 t}\right) \frac{d t}{2 t} \\
R_{\nu, l-1}((r, \tau ; z) & =-(r \tau)^{-\frac{n-2}{2}} \int_{0}^{\infty} e^{i \frac{\rho}{t}-i \frac{\pi \nu}{2}} \mathcal{O}_{l-1}\left(e^{i r \tau z t}\right) J_{\nu}\left(\frac{1}{2 t}\right) \frac{d t}{2 t} .
\end{aligned}
$$

Here and in the following, $\mathcal{O}_{N}(g(s))$ denotes the remainder in Taylor expansion of $g$ up to the $N$-th order:

$$
\mathcal{O}_{N}(g(s))=g(s)-\sum_{j=0}^{N} \frac{g^{(k)}(0)}{k !} s^{k}=\frac{1}{N !} \int_{0}^{1}(1-\theta)^{N} s^{N+1} g^{(N+1)}(s \theta) d \theta .
$$

If $l=0$ and $0 \leq \nu<1$, we just set $R_{\nu, l-1}(z)=K_{\nu}(z)$. Split $R_{\nu, l-1}(z)$ into two pieces

$$
R_{\nu, l-1}(z)=R_{\nu, l-1,1}(z)+R_{\nu, l-1,2}(z)
$$

with $R_{\nu, l-1,1}(z)$ defined by the integral for $\left.\left.t \in\right] 0,1\right]$ in (2.10). It is clear that $R_{\nu, l-1,1}(z)$ has the following asymptotic expansion for any $N$

$$
R_{\nu, l-1,1}(r, \tau ; z)=\sum_{j=l}^{N} z^{j} K_{\nu, 1, j}(r, \tau)+R_{\nu, N, 1}(r, \tau ; z),
$$

where

$$
K_{\nu, 1, j}(r, \tau)=-(r \tau)^{-\frac{n-2}{2}+j} \int_{0}^{1} e^{i \frac{\rho}{t}-i \frac{\pi \nu}{2}} \frac{(i t)^{j}}{j !} J_{\nu}\left(\frac{1}{2 t}\right) \frac{d t}{2 t}
$$


and

$$
R_{\nu, N, 1}(r, \tau ; z)=-(r \tau)^{-\frac{n-2}{2}} \int_{0}^{1} e^{i \frac{\rho}{t}-i \frac{\pi \nu}{2}} J_{\nu}\left(\frac{1}{2 t}\right) \mathcal{O}_{N}\left(e^{i z r \tau t}\right) \frac{d t}{2 t} .
$$

The asymptotic expansion of $R_{\nu, l-1,2}(z)$ can be deduced from Lemma A.1. Let us begin with the case $l=0$ and $\nu \in\left[0,1\left[\right.\right.$. Then, $K_{\nu, 2}(z) \equiv R_{\nu, l-1,2}(z)$ can be written in the form

$$
K_{\nu, 2}(r, \tau ; z)=\int_{1}^{\infty} e^{i z r \tau t} t^{-1-\nu} f\left(\frac{1}{t} ; r, \tau, \nu\right) d t
$$

where

$$
f(s ; r, \tau, \nu)=D_{\nu}(r, \tau) \int_{-1}^{1} e^{i(\rho+\theta / 2) s}\left(1-\theta^{2}\right)^{\nu-1 / 2} d \theta, \quad \nu \geq 0,
$$

with

$$
D_{\nu}(r, \tau)=a_{\nu}(r \tau)^{-\frac{n-2}{2}}, \quad a_{\nu}=-\frac{e^{-i \pi \nu / 2}}{2^{2 \nu+1} \pi^{1 / 2} \Gamma(\nu+1 / 2)} .
$$

Write $f(s ; r, \tau, \nu)=\sum_{j=0}^{\infty} s^{j} f_{j}(r, \tau, \nu), s \in \mathbb{R}$, where

$$
f_{j}(r, \tau, \nu)=(r \tau)^{-\frac{n-2}{2}} P_{j, \nu}(\rho)
$$

with $P_{j, \nu}(\rho)$ a polynomial in $\rho$ of degree $j$ :

$$
P_{j, \nu}(\rho)=\frac{i^{j} a_{\nu}}{j !} \int_{-1}^{1}(\rho+\theta / 2)^{j}\left(1-\theta^{2}\right)^{\nu-1 / 2} d \theta .
$$

In particular,

$$
\begin{aligned}
& f_{0}(r, \tau, \nu)=d_{\nu}(r \tau)^{-\frac{n-2}{2}}, \quad d_{\nu}=-\frac{e^{-i \pi \nu / 2}}{2^{2 \nu+1} \Gamma(\nu+1)} \\
& f_{1}(r, \tau, \nu)=i d_{\nu}(r \tau)^{-\frac{n-2}{2}} \rho .
\end{aligned}
$$

If $\nu \in[0,1[$, Lemma A.1 can be applied to obtain an asymptotic expansion for $K_{\nu, 2}(z)$. When $0<\nu<1$, (a) of Lemma A.1 gives

$$
K_{\nu, 2}(z)=\sum_{j=0}^{N} K_{\nu, 2, j}(r, \tau) z^{j}+z^{\nu} \sum_{j=0}^{N} z^{j} G_{\nu, j}(r, \tau)+R_{\nu, N, 2}(r, \tau, z)
$$


where

$$
\begin{aligned}
F_{\nu, 2, j}(r, \tau) & =(r \tau)^{j} C_{\nu, j} \\
G_{\nu, j}(r, \tau) & =(r \tau)^{j+\nu} b_{\nu, j} f_{j} \\
R_{\nu, N, 2}(r, \tau, z) & =\tilde{R}_{\nu, N, 2}(r \tau z)
\end{aligned}
$$

When $\nu=0$, we derive from (b) of Lemma A.1 that

$$
K_{0,2}(z)=\sum_{j=0}^{N} K_{0,2, j}(r, \tau) z^{j}+\ln z \sum_{j=0}^{N} z^{j} G_{0, j}(r, \tau)+R_{0, N, 2}(r, \tau, z)
$$

where

$$
\begin{aligned}
K_{0,2, j}(r, \tau) & =(r \tau)^{j}\left(C_{0, j}-\frac{i^{j} f_{j}}{j !} \ln (r \tau)\right), 0 \leq j \leq N, \\
G_{0, j}(r, \tau) & =-(i r \tau)^{j} \frac{f_{j}}{j !}, \quad 0 \leq j \leq N, \\
R_{0, N, 2}(r, \tau, z) & =(i z r \tau)^{N+1} f_{N+1} b_{N+1}+\tilde{R}_{0, N, 2}(r \tau z) .
\end{aligned}
$$

Here all quantities are defined as in Lemma A.1 with $f(s)=f(s ; r, \tau, \nu)$ and $f_{j}=f_{j}(r, \tau, \nu)$.

In the case $l \geq 1$, inserting the integral remainder formula of $\mathcal{O}_{l-1}\left(e^{i r \tau z t}\right)$ into (2.10), we can then apply Lemma A.1 to obtain the expansion of $R_{\nu, l-1,2}(z)$. Summing up, we have proved the following

Proposition 2.1 Let $\nu \in \sigma_{\infty}$ and $l \in \mathbb{N}$ with $l \leq \nu<l+1$. Set $\nu^{\prime}=\nu-l \in$ $[0,1[$.

(a). If $l<\nu<l+1$, one has

$$
\left(Q_{\nu}-z\right)^{-1}=\sum_{j=0}^{N} z^{j} F_{\nu, j}+z^{\nu^{\prime}} \sum_{j=l}^{N-1} z^{j} G_{\nu, j}+R_{\nu, N}(z)
$$


with $F_{\nu, j}$ given by (2.9) for $0 \leq j \leq l$ and for $l+1 \leq j \leq N$,

$$
\begin{aligned}
F_{\nu, j}= & \frac{i^{l}(j-l) !}{j !}(r \tau)^{j} C_{\nu^{\prime}, j-l}+\frac{(i r \tau)^{j}}{j !} \int_{0}^{1} t^{j-\nu-1} f\left(\frac{1}{t} ; r, \tau, \nu\right) d t \\
G_{\nu, j}= & (r \tau)^{j+\nu^{\prime}} b_{\nu^{\prime}, j} f_{j-l}, \quad l \leq j \leq N \\
R_{\nu, N}(z)= & z^{\nu^{\prime}+N} G_{\nu, N}+\frac{(i r \tau z)^{l}}{(l-1) !} \int_{0}^{1}(1-\theta)^{l-1}\left(\tilde{R}_{\nu^{\prime}, N-l, 2}(\theta z r \tau)\right. \\
& \left.+\int_{0}^{1} \mathcal{O}_{N-l}\left(e^{i t \theta z r \tau}\right) t^{l-1-\nu} f\left(\frac{1}{t} ; r, \tau, \nu\right) d t\right) d \theta
\end{aligned}
$$

When $l=0$, the integral in $\theta$ is absent.

(b). If $\nu=l \in \mathbb{N}$, then,

$$
\left(Q_{\nu}-z\right)^{-1}=\sum_{j=0}^{N} z^{j} F_{\nu, j}+\ln z \sum_{j=l}^{N} z^{j} G_{\nu, j}+R_{\nu, N}(z)
$$

with $F_{\nu, j}$ given by (2.9) for $0 \leq j \leq l-1$ and

$$
\begin{aligned}
F_{\nu, j}= & (r \tau)^{j}\left\{\frac{i^{l}(j-l) !}{j !} C_{0, j-l}-\ln (r \tau) \frac{i^{j} f_{j-l}}{j !}-c_{l, j} f_{j-l}\right\} \\
& +\frac{(i r \tau)^{j}}{j !} \int_{0}^{1} t^{j-l-1} f\left(\frac{1}{t} ; r, \tau, \nu\right) d t, \quad l \leq j \leq N, \\
G_{\nu, j}= & -(i r \tau)^{j} \frac{f_{j-l}}{j !}, \quad l \leq j \leq N, \\
R_{0, N}(z)= & (i z r \tau)^{N+1} f_{N+1} b_{N+1}+\tilde{R}_{0, N, 2}(z r \tau) \\
& +\int_{0}^{1} \mathcal{O}_{N}\left(e^{i t z r \tau}\right) t^{-1} f\left(\frac{1}{t} ; r, \tau, \nu\right) d t \\
& (i r \tau z)^{N+1}(N-l+1) ! \\
R_{l, N}(z) & (N+33) \\
& \left.+\int_{0}^{1} \mathcal{O}_{N-l}\left(e^{i t \theta z r \tau}\right) t^{-1} f\left(\frac{1}{t} ; r, \tau, \nu\right) d t\right) d \theta
\end{aligned}
$$

for $\nu=l \geq 1$. Here $c_{0, j}=0$ for all $j$ and

$$
c_{l, j}=-\frac{i^{j}}{(l-1) !(j-l) !} \int_{0}^{1}(1-\theta)^{l-1} \theta^{j-l} \ln \theta d \theta, \quad l \geq 1, j \geq l .
$$

$C_{\nu^{\prime}, j}=C_{\nu^{\prime}, j}(r, \tau)$ and $b_{\nu^{\prime}, j} \in \mathbb{C}$ are defined in Lemma $A .1$ for $0 \leq \nu^{\prime}<1$ with $f\left(\frac{1}{t}\right)$ replaced by $f\left(\frac{1}{t} ; r, \tau, \nu\right)$. 
Let $L^{2, s}$ denote the weighted $L^{2}$-space $L^{2}\left(\mathbb{R}_{+},(1+r)^{s} r^{n-1} d r\right)$ and $\mathcal{L}\left(0, s ; 0, s^{\prime}\right)$ the space of bounded linear operators from $L^{2, s}$ to $L^{2, s^{\prime}}$. In the later, we use the same notation for weighted $L^{2}$-spaces over $\mathbb{R}^{n}$.

Lemma 2.2 Let $\nu \geq 0$. Let $[\nu]$ denote the integral part of $\nu$ and $\nu^{\prime}=\nu-[\nu]$. Let $F_{\nu, j}, G_{\nu, j}, R_{\nu, N}(z)$ be defined as before for different values of $\nu$ and $j$.

$$
\begin{aligned}
& F_{\nu, j} \in \mathcal{L}(0, s ; 0,-s), \quad s>j+1, \text { for } j<\nu ; \quad s>2 j-[\nu]+1, \text { for } j \geq \nu \\
& G_{\nu, j} \in \mathcal{L}(0, s ; 0,-s), \quad s>2 j-[\nu]+1+\nu^{\prime}, j \geq[\nu] \\
& R_{\nu, N}(z)=O\left(|z|^{N+\epsilon}\right) \in \mathcal{L}(0, s ; 0,-s), \quad s>2 N-\nu+1,
\end{aligned}
$$

Proof. Let $\nu \notin \mathbb{N}$. When $j<\nu, F_{\nu, j}$ given by (2.9) can be bounded by

$$
\left|F_{\nu, j}(r, \tau)\right| \leq(r \tau)^{-\frac{n-2}{2}+j} \frac{1}{j !} \int_{0}^{\infty} t^{j}\left|J_{\nu}\left(\frac{1}{2 t}\right)\right| \frac{d t}{2 t} .
$$

By the asymptotic behavior of Bessel functions, the above integral is convergent. One can show that the convergence is uniform in $\nu \in \sigma_{\infty}$ for each $j$. In fact, from the properties of Bessel functions (see [20, 26]), it follows that

$$
\begin{aligned}
\int_{0}^{1} \tau^{-j-1}\left|J_{\nu}(\tau)\right| d \tau & \leq \frac{1}{2^{\nu}(\nu-j) \Gamma(\nu+1)} \\
\int_{1}^{\infty} \tau^{-j-1}\left|J_{\nu}(\tau)\right| d \tau & \leq c_{\delta} \frac{\Gamma(\nu / 2-j / 2+\delta / 2)}{2^{j+1} \Gamma(\nu / 2+j / 2+1-\delta / 2)}
\end{aligned}
$$

Here $0<\delta<\nu-j$ is small. It follows that

$$
\left|F_{\nu, j}(r, \tau)\right| \leq c_{j}(r \tau)^{-\frac{n-2}{2}+j}, \quad j<\nu,
$$

$c_{j}$ is independent of $\nu \in \sigma_{\infty}$. If $s>j+1,(1+r)^{-s}(r \tau)^{-\frac{n-2}{2}+j}(1+\tau)^{-s}$ defines a Hilbert-Schmidt operator on $L^{2}\left(\mathbb{R}_{+} ; r^{n-1} d r\right)$. It follows that

$$
\left\|F_{\nu, j}\right\|_{\mathcal{L}(0, s ; 0-s)} \leq c(j, s), \quad \forall \nu>j, \nu \in \sigma_{\infty}
$$

where $c(j, s)$ is a constant independent of $\nu$. 
For $j \geq \nu, F_{\nu, j}$ and $G_{\nu, j}$ depend on $f_{0}, \cdots, f_{j-[\nu]}$ with $f_{k}(r, \tau, \nu)=$ $(r \tau)^{-\frac{n-2}{2}} P_{k, \nu}(\rho)$ where $P_{k, \nu}$ is a polynomial of degree $k$ in $\rho$. Since for $j \geq k$

$$
(r \tau)^{j}(r \tau)^{-\frac{n-2}{2}} \rho^{k}=(r \tau)^{-\frac{n-2}{2}+j-k}\left(\left(r^{2}+\tau^{2}\right) / 4\right)^{k}
$$

it is easy to see that if $s>k+j+1,(1+x)^{-s}(r \tau)^{j} f_{k}(r, \tau, \nu)(1+y)^{-s}$ defines a Hilbert-Schmidt operator on $L^{2}\left(\mathbb{R}_{+} ; r^{n-1} d r\right)$, so it belongs to $\mathcal{L}(0, s ; 0,-s)$. Since $\mathcal{O}_{j}\left(f\left(\frac{1}{t} ; r, \tau, \nu\right)\right)$ can be bounded by

$$
c_{\epsilon}(r \tau)^{-\frac{n-2}{2}}\left(\frac{\rho}{t}\right)^{j+\epsilon}, \quad \forall \epsilon \in[0,1]
$$

this shows that $(r \tau)^{j} C_{\nu^{\prime}, j-l}$ defines an operator in $\mathcal{L}(0, s ; 0,-s)$ for $s>2 j-l+1$. Therefore, $F_{\nu, j} \in \mathcal{L}(0, s ; 0,-s)$ for $s>2 j-l+1$. The continuity of $G_{\nu, j}$ is evident.

To estimate the remainder, remark that if $\nu \in \mathbb{N}$, one has $R_{\nu, N}(z)=$ $O\left(|z|^{N+1}\right)$ in $\mathcal{L}(0, s ; 0,-s)$ for $s>2 N-\nu+3$. Note that $R_{\nu, N}(z)$ can be expressed in terms of $R_{\nu, N-1}(z), F_{\nu, N}$ and $G_{\nu, N}$. It follows that $R_{\nu, N}(z)=O\left(|z|^{N}|\ln z|\right)$ in $\mathcal{L}(0, s ; 0,-s)$ for $s>2 N-\nu+1$. A complex interpolation gives the desired estimate in the case $\nu \in \mathbb{N}$.

If $\nu \notin \mathbb{N}, \mathcal{O}_{N-l}\left(f\left(\frac{1}{t} ; r, \tau, \nu\right)\right)$ can be bounded by $c(r \tau)^{-\frac{n-2}{2}}\left(\frac{\rho}{t}\right)^{N-l+\epsilon}$ for any $\epsilon \in[0,1]$. We derive that for $s>2 N-[\nu]+\nu^{\prime}+1, R_{\nu, N}(z)=O\left(|z|^{N+\nu^{\prime}}\right)$ in $\mathcal{L}(1,-s ;-1, s)$. In terms of $R_{\nu, N-1}(z), F_{\nu, N}$ and $G_{\nu, N-1}$, we can also estimate that $R_{\nu, N}(z)=O\left(|z|^{N-1+\nu^{\prime}}\right)$ in $\mathcal{L}(1,-s ;-1, s)$ for $s>2 N-[\nu]+\nu^{\prime}-1$. A complex interpolation gives that $R_{\nu, N}(z)=O\left(|z|^{N+\epsilon}\right)$ in $\mathcal{L}(1,-s ;-1, s)$, if $s>2 N+1-[\nu]-\nu^{\prime}=2 N+1-\nu$.

Define for $\nu \in \sigma_{\infty}$

$$
z_{\nu}=\left\{\begin{array}{cc}
z^{\nu^{\prime}}, & \text { if } \nu \notin \mathbb{N} \\
z \ln z, & \text { if } \nu \in \mathbb{N}
\end{array}\right.
$$

Let $\sigma_{N}=\sigma_{\infty} \cap[0, N]$. For $\nu>0$, let $[\nu]_{-}$be the largest integer strictly less than $\nu$. When $\nu=0$, set $[\nu]_{-}=0$. Define $\delta_{\nu}$ by $\delta_{\nu}=1$, if $\nu \in \sigma_{\infty} \cap \mathbb{N} ; 0$, otherwise. One has $[\nu]=[\nu]_{-}+\delta_{\nu}$. 
Theorem 2.3 The following asymptotic expansion holds for $z$ near 0 with $\Im z>$ 0 .

$$
R_{0}(z)=\delta_{0} \ln z G_{0,0}+\sum_{j=0}^{N} z^{j} F_{j}+\sum_{\nu \in \sigma_{N}} z_{\nu} \sum_{j=[\nu]_{-}}^{N-1} z^{j} G_{\nu, j+\delta_{\nu}} \pi_{\nu}+R_{0}^{(N)}(z),(2
$$

in $\mathcal{L}(-1, s ; 1,-s), s>2 N+1$. Here

$$
\begin{aligned}
F_{j} & =\sum_{\nu \in \sigma_{\infty}} F_{\nu, j} \pi_{\nu} \in \mathcal{L}(-1, s ; 1,-s), \quad s>2 j+1 \\
R_{0}^{(N)}(z) & =O\left(|z|^{N+\epsilon}\right) \in \mathcal{L}(-1, s ; 1,-s), \quad s>2 N+1, \quad \epsilon>0 .
\end{aligned}
$$

Proof. Note that for fixed $j$ and $N$, apart from a finite number of $\nu, F_{\nu, j}$ and $R_{\nu, N}(z)$ are given by (2.9) and (2.10), respectively. So we can use the properties of Bessel functions to prove as in Lemma 2.2 that they are bounded in $\mathcal{L}(0, s ; 0,-s)$ for appropriate $s$, uniformly w.r.t. $\nu \in \sigma_{\infty}$. The result in $\mathcal{L}(-1, s ; 1,-s)$ follows from the ellipticity of $P_{0}$.

Remark 2.4 (a). It is proved in [11] that under the assumption $0 \notin \sigma_{\infty}, R_{0}(z)$ is uniformly bounded in $\mathcal{L}(-1, s ; 1,-s), s>1$, and under the assumption $[0,1] \cap$ $\sigma_{\infty}=\emptyset$, it is $C^{1}$ up to the real axis in $\mathcal{L}(-1, s ; 1,-s), s>2$.

(b). Assume that $0 \notin \sigma_{\infty}$. If $s>1$, Theorem 2.3 gives

$$
R_{0}(z)=F_{0}+O\left(|z|^{\epsilon}\right)
$$

in $\mathcal{L}(-1, s ; 1,-s)$ for some $\epsilon>0$. If $s>3$, then,

$$
R_{0}(z)=F_{0}+z F_{1}+\sum_{\nu \in \sigma_{1}} z_{\nu} G_{\nu, \delta_{\nu}} \pi_{\nu}+O\left(|z|^{1+\epsilon}\right)
$$

in $\mathcal{L}(-1, s ; 1,-s)$. Here

$$
\begin{aligned}
F_{0} & \in \mathcal{L}(-1, s ; 1,-s), \quad s>1, \\
F_{1} & \in \mathcal{L}(-1, s ; 1,-s), \quad s>3, \quad\left(s>2, \quad \text { if } \sigma_{\infty} \cap[0,1]=\emptyset\right), \\
G_{\nu, \delta_{\nu}} & =c_{\nu}(r \tau)^{-\frac{n-2}{2}+\nu}, \quad 0<\nu \leq 1, \\
c_{\nu} & =-\frac{e^{-i \pi \nu} \Gamma(1-\nu)}{\nu 2^{2 \nu+1} \Gamma(\nu+1)}, \quad 0<\nu<1, \quad c_{1}=\frac{1}{8} .
\end{aligned}
$$


(c). The remainder estimates can be improved with the help of information on $\sigma_{1}$. Making use of the kernel of the remainder $R_{\nu, 0}(z)$, we can show that if $\nu>1$,

$$
\left(Q_{\nu}-z\right)^{-1}=F_{\nu, 0}+O\left(|z|^{\delta}\right)
$$

in $\mathcal{L}(-1, s ; 1,-s), s>1+\delta, 0<\delta \leq 1$, and if $0<\nu \leq 1$,

$$
\left(Q_{\nu}-z\right)^{-1}=F_{\nu, 0}+z_{\nu} G_{\nu, \delta_{\nu}}+O\left(|z|^{\delta}\right)
$$

in $\mathcal{L}(-1, s ; 1,-s), s>1+2 \delta-\nu, \nu \leq \delta \leq 1$. Let $\nu_{0}=\min \left\{\nu \in \sigma_{1}\right\}>0$ and $\nu_{M}=\max \left\{\nu \in \sigma_{1}\right\} \leq 1$. Then,

$$
R_{0}(z)=F_{0}+\sum_{0<\nu \leq 1} z_{\nu} G_{\nu, \delta_{\nu}} \pi_{\nu}+O\left(|z|^{\delta}\right)
$$

for $\nu_{M} \leq \delta \leq 1$ and $s>1+2 \delta-\nu_{0}$.

\section{Long-time expansion of wave functions of $P_{0}$}

Consider the Schrödinger equation

$$
\left\{\begin{aligned}
i \frac{\partial}{\partial t} u(t) & =P_{0} u(t) \\
u(0) & =u_{0}
\end{aligned}\right.
$$

with $u_{0} \in L^{2}\left(M_{0}\right)$. Let

$$
U_{0}(t)=e^{-i t P_{0}}, \quad t \in \mathbb{R}
$$

Then,

$$
U_{0}(t)=\frac{1}{2 i \pi} \int_{\mathbb{R}} e^{-i t \lambda} R_{0}(\lambda+i 0) d \lambda, \quad t>0 .
$$

The long-time expansion of solutions to the Schrödinger equation associated with $P_{0}$ can be easily deduced from Theorem 2.3.

Theorem 3.1 One has in $\mathcal{L}(0, s ; 0,-s)$ with $s>2 N+1$

$$
U_{0}(t)=\sum_{\nu \in \sigma_{N}} \sum_{j=[\nu]}^{N-1+\delta_{\nu}} t^{-j-1-\nu^{\prime}} a_{\nu, j} G_{\nu, j} \pi_{\nu}+O\left(|t|^{-N-1-\epsilon}\right) .
$$


Here

$$
\begin{aligned}
G_{\nu, j}(r, \tau) & = \begin{cases}b_{\nu^{\prime}, j}(r \tau)^{j+\nu^{\prime}} f_{j-[\nu]}\left(r, \tau ; \nu^{\prime}\right), & \nu \notin \mathbb{N}, \\
-\frac{(i r \tau)^{j}}{j !} f_{j-[\nu]}(r, \tau ; 0), & \nu \in \mathbb{N} .\end{cases} \\
a_{\nu, j} & = \begin{cases}\frac{i}{\pi}(-i)^{j} \sin (\nu \pi) e^{i \pi \nu / 2} \Gamma(\nu+j+1), & \nu \notin \mathbb{N} \\
i(-i)^{j+\nu}(\nu+j) !, & \nu \in \mathbb{N} .\end{cases}
\end{aligned}
$$

$f_{j-[\nu]}\left(r, \tau ; \nu^{\prime}\right)$ is given by (2.16) and $b_{\nu^{\prime}, j}$ by Lemma A.1.

Proof. Let $A$ denote the generator of the dilation group: $A=i^{-1}\left(r \partial_{r}+\partial_{r} r\right)$. Since $M_{0}=\mathbb{R}_{+} \times \Sigma$, one has

$$
i\left[P_{0}, A\right]=2 P_{0}, \quad \underbrace{i\left[\cdots i\left[P_{0}, A\right], \cdots, A\right]}_{k \text { times }}=2^{k} P_{0}, \quad k \geq 2,
$$

in the sense of forms. The Mourre's method with multiple commutator estimates can be applied. It follows that for $k+1 / 2<s \leq k+1, k \in \mathbb{N}$,

$$
\left\|\langle x\rangle^{-s} \frac{d^{k}}{d \lambda^{k}} R_{0}(\lambda+i 0)\langle x\rangle^{-s}\right\| \leq C_{k, s} \lambda^{-(k+1) / 2}, \quad \lambda \geq \epsilon>0 .
$$

Let $\chi \in C_{0}^{\infty}(\mathbb{R})$ with support in a small neighborhood of 0 and equal to 1 near 0. By the Fourier transform, one obtains from (3.6) that

$$
U_{0}(t)\left(1-\chi\left(P_{0}\right)\right)=O\left(t^{-2 N}\right), \quad t \rightarrow+\infty
$$

in $\mathcal{L}(0, s ; 0,-s)$ for $s>2 N+1$. It remains to apply Theorem 2.3 and Lemma A.2 to $U_{0}(t) \chi\left(P_{0}\right)$ in order to obtain $(3.3)$.

Example. Let $M_{0}=\mathbb{R}_{+} \times \Sigma$, where $\Sigma$ is the unit circle deprived of one point: $\boldsymbol{\Sigma}=\mathbb{S}^{1} \backslash\{(1,0)\} \subset \mathbb{R}^{2}$. Let $h_{a}=a^{2}(d \theta)^{2}, a>0$, on $\boldsymbol{\Sigma}$ and $-\Delta_{g_{0}}$ the Dirichlet realization of $-\Delta_{g_{0}}$ on $M_{0}$ with $g_{0}=d r^{2}+a^{2}(d \theta)^{2}$. Then,

$$
e^{i t \Delta_{g_{0}}} \simeq C t^{-\frac{a}{2}-1}, \quad t \rightarrow \infty
$$

In particular, if $a=2 \pi$ (so that the length of the unit circle is equal to one in the metric $h_{2 \pi}$ ), then

$$
e^{i t \Delta_{g_{0}}} \simeq C t^{-\pi-1}, \quad t \rightarrow \infty
$$

Here $C$ is some operator of finite rank. 
Remark 3.2 In [7], global Strichartz estimates are proved for Schrödinger and wave equations associated to $-\Delta+\frac{a}{r^{2}}$, where $-\Delta$ is the Laplacian on $\mathbb{R}^{n}$ and $a$ is a constant with $a>-(n-2)^{2} / 4$. More precisely, they proved for $u(t)=$ $e^{-i t\left(-\Delta+\frac{a}{r^{2}}\right)} f, f \in L^{2}$, that

$$
\|u\|_{L_{t}^{p}\left(L_{x}^{q}\right)} \leq C(p, n, a)\|f\|_{L^{2}}
$$

where $\frac{2}{p}+\frac{n}{q}=\frac{n}{2}, p \geq 2$ and $(n, p) \neq(2,2)$. We believe that similar estimates hold for $P_{0}=-\Delta_{g_{0}}+\frac{q(\theta)}{r^{2}}$ on $\mathbb{R}_{+} \times \boldsymbol{\Sigma}$ under the condition $0 \notin \sigma_{1}$.

\section{Eigenfunctions and resonant states at zero}

Let $M$ be a Riemannian manifold which outside some compact is isometric to $\mathbb{R}_{+} \times \Sigma$. Consider the perturbation of $P_{0}$ in the form

$$
P=-\Delta_{g}+\frac{q(\theta)}{r^{2}}+V_{0}(x)
$$

on $M$, where $x=r \theta$ is polar coordinates around some point $x_{0} \in M, g$ is a Riemannian metric on $M$ and $V_{0}$ is real function such that

$$
g-g_{0}=O\left(|x|^{-\rho_{0}}\right), \quad V_{0}(x)=O\left(|x|^{-\rho_{0}}\right)
$$

for some $\rho_{0}>2$ as $|x|=d\left(x, x_{0}\right) \rightarrow \infty . g$ is assumed to be $C^{2}$ and $V_{0}$ bounded. We also assume that $P$ is self-adjoint as form defined on $H_{0}^{1}$. A sufficient condition is that

$$
\frac{q(\theta)}{r^{2}} \geq-a\left(-\Delta_{g}\right)-b
$$

for some $0 \leq a<1$ and $b \in \mathbb{R}_{+}$. Let $K$ be a compact of $M$ such that $M_{0}=M \backslash K$ is isometric to $\mathbb{R}_{+} \times \boldsymbol{\Sigma}$. On $M_{0}, P$ can be written as

$$
P=P_{0}+V, \quad V=-\Delta_{g}+\Delta_{g_{0}}+V_{0}(x) .
$$

Remark that the extension by 0 of $v \in H_{0}^{1}\left(M_{0}\right)$ is in $H_{0}^{1}(M)$, and the restriction of $w \in H^{-1}(M)$ to $M_{0}$ belongs to $H^{-1}(M)$. 
Definition Set $\mathcal{N}=\left\{u ; P u=0, u \in H_{0}^{1,-s}, \forall s>1\right\}$. A function $u \in \mathcal{N} \backslash L^{2}$ is called a resonant state of $P$ at zero.

The asymptotic expansion of solutions to elliptic equations on manifold with conical singularities is studied in $[16,17]$. To establish the existence of an asymptotic expansion of the resolvent $R(z)=(P-z)^{-1}([25])$, it is crucial for us to characterize the resonant states and to distinguish them from eigenfunctions.

Theorem 4.1 Assume $\rho_{0}>3$ and that $0 \notin \sigma_{1}$. Let $u \in \mathcal{N}$. Then,

$$
u(r \theta)=\sum_{0<\nu \leq 1} \sum_{j=1}^{n_{\nu}}-\frac{1}{2 \nu}<V u,|y|^{-\frac{n-2}{2}+\nu} \varphi_{\nu}^{(j)}>\frac{\varphi_{\nu}^{(j)}(\theta)}{r^{\frac{n-2}{2}+\nu}}+v
$$

where $v \in L^{2}$, and $<\cdot, \cdot>$ is the scalar product in $L^{2}\left(M_{0} ; d v_{g_{0}}\right)$. In particular,

$$
u \in L^{2} \Longleftrightarrow<V u,|y|^{-\frac{n-2}{2}+\nu} \varphi_{\nu}^{(j)}>=0, \quad \forall \nu \in \sigma_{1}, 1 \leq j \leq n_{\nu} .
$$

Let $\mathcal{C}$ denote the linear span of all vectors of the form

$$
c(u)=\left(\frac{1}{2 \nu}<V u,-|y|^{-\frac{n-2}{2}+\nu} \varphi_{\nu}^{(j)}>; \nu \in \sigma_{1}, 1 \leq j \leq n_{\nu}\right) \in \mathbb{C}^{\kappa},
$$

with $u \in \mathcal{N}, \kappa=\sum_{\nu \in \sigma_{1}} n_{\nu}$. Then,

$$
\operatorname{dim}\left(\mathcal{N} /\left(\operatorname{ker}_{L^{2}} P\right)\right)=\operatorname{dim} \mathcal{C}
$$

Proof. We only need to work with $r$ large. For $u \in \mathcal{N}$, set $u=\sum_{0<\nu \leq 1} \sum_{j=1}^{n_{\nu}} u_{\nu, j}+$ $u^{\prime}$, where $u^{\prime}=\pi^{\prime} u$ with $\pi^{\prime}=\sum_{\nu>1} \pi_{\nu}$ and $u_{\nu, j}=\left(u, \varphi_{\nu}^{(j)}\right)_{\Sigma}$. Then in $M_{0}$, one has

$$
P_{0} u^{\prime}=-\pi^{\prime}(V u)
$$

In the cylindrical coordinates $(t, \theta)\left(x=r \theta, r=e^{t}\right)$, one has

$$
|x|^{2+\tau} P_{0}|x|^{-\tau}=-\partial_{t}^{2}-(n-2-2 \tau) \partial_{t}+\tau(n-2-\tau)+\Lambda
$$

with $\Lambda=-\Delta_{h}+q(\theta)$. Taking $\tau=\frac{n-2}{2}$, we obtain

$$
|x|^{2+\frac{n-2}{2}} P_{0}|x|^{-\frac{n-2}{2}}=-\partial_{t}^{2}+\frac{(n-2)^{2}}{4}+\Lambda
$$


Let $u_{\nu, j}=\left(u, \varphi_{\nu}^{(j)}\right)_{\Sigma},(V u)_{\nu, j}=\left(V u, \varphi_{\nu}^{(j)}\right)_{\Sigma}$. Let $v_{\nu, j}=|x|^{\frac{n-2}{2}} u_{\nu, j}$. Then,

$$
\left(-\partial_{t}^{2}+\nu^{2}\right) v_{\nu, j}=-e^{\frac{n+2}{2} t}(V u)_{\nu, j}
$$

For the equation $\left(-\partial_{t}^{2}+\nu^{2}\right) f=g$, we have the following Agmon-type energy estimate

$$
\left\|e^{\nu^{\prime}|t|} f\right\| \leq C_{\epsilon}\left\|e^{\nu^{\prime}|t|} g\right\|, \quad \forall \quad f, g \in C_{0}^{\infty},
$$

where $\nu^{\prime}=\nu-\epsilon, \epsilon>0$ can be arbitrarily small and $C_{\epsilon}$ is independent of $\nu, f$ and $g$, and is uniformly bounded if $\epsilon$ is away from 0. By a density argument, we can show that if $e^{(\nu-\epsilon)|t|+\frac{n+2}{2} t}(V u)_{\nu, j}\left(e^{t}\right)$ is in $L^{2}(\mathbb{R} ; d t)$, then $e^{(\nu-\epsilon)|t|} v_{\nu, j}\left(e^{t}\right)$ is in $L^{2}(\mathbb{R} ; d t)$ and

$$
\left\|e^{(\nu-\epsilon)|t|} v_{\nu, j}\right\| \leq C_{\epsilon}\left\|e^{(\nu-\epsilon)|t|+\frac{n+2}{2} t}(V u)_{\nu, j}\right\|
$$

In $L^{2}\left(\mathbb{R}_{+}, r^{n-1} d r\right)$, we have

$$
\begin{gathered}
\left\|r^{\nu-1-\epsilon} u_{\nu, j}\right\| \leq C_{\epsilon}\left\|r^{\nu+1-\epsilon}(V u)_{\nu, j}\right\| \\
\text { if } r^{\nu+1-\epsilon}(V u)_{\nu, j} \in L^{2}\left(\mathbb{R}_{+}, r^{n-1} d r\right) \text {. Since } \rho_{0}>3,(V u)_{\nu, j} \in L^{2, \rho_{0}-1-\epsilon}\left(\mathbb{R}_{+}, r^{n-1} d r\right) .
\end{gathered}
$$

Take

$$
1<\nu^{\prime}<\min \left\{\rho_{0}-2, \min \left\{\nu \in \sigma_{\infty} \backslash \sigma_{1}\right\}\right\}
$$

We obtain

$$
\left\|r^{\nu^{\prime}-1} u_{\nu, j}\right\| \leq C\left\|r^{\nu^{\prime}+1}(V u)_{\nu, j}\right\|
$$

uniformly in $\nu>1$. Summing up the series in $(\nu, j)$ with $\nu>1$, one sees that $u^{\prime} \in L^{2}$ and that $\left\|\langle r\rangle^{\nu^{\prime}-1} u^{\prime}\right\| \leq C\left\|\langle r\rangle^{1+\nu^{\prime}} \pi^{\prime}(V u)\right\|$.

For $0<\nu \leq 1$, we seek the leading asymptotics of $u_{\nu, j}(r)$ as $r \rightarrow \infty$. We want to deduce from (4.9) that

$$
u_{\nu, j}(r)=-\frac{1}{2 \nu} \int_{0}^{\infty} \tau^{-\frac{n-2}{2}+\nu}(V u)_{\nu, j}(\tau) \tau^{n-1} d \tau r^{-\frac{n-2}{2}-\nu}+u_{\nu, j}^{\prime},
$$

with $u_{\nu, j}^{\prime} \in L^{2}\left(\mathbb{R}_{+} ; r^{n-1} d r\right)$. By (4.9), $v_{\nu, j}$ can be represented as

$$
v_{\nu, j}\left(e^{t}\right)=C_{+} e^{\nu t}+C_{-} e^{-\nu t}-\frac{1}{2 \nu} \int_{\mathbb{R}} e^{-\nu|t-s|} e^{\frac{n+2}{2} s}(V u)_{\nu, j}\left(e^{s}\right) d s
$$


Since $u_{\nu, j}=-F_{\nu, 0}(V u)_{\nu, j}$, we see that $\left|u_{\nu, j}(r)\right| \leq C r^{-\frac{n-2}{2}}$ for all $r>0$. This shows $C_{+}=C_{-}=0$. Now set $v_{\nu, j}\left(e^{t}\right)=v_{\nu, j}^{(0)}+v_{\nu, j}^{(1)}+v_{\nu, j}^{(2)}$ with

$$
\begin{aligned}
& v_{\nu, j}^{(0)}\left(e^{t}\right)=-\frac{1}{2 \nu} \int_{-\infty}^{\infty} e^{-\nu(t-s)} e^{\frac{n+2}{2} s}(V u)_{\nu, j}\left(e^{s}\right) d s \\
& v_{\nu, j}^{(1)}\left(e^{t}\right)=\frac{1}{2 \nu} \int_{t}^{\infty} e^{-\nu(t-s)} e^{\frac{n+2}{2} s}(V u)_{\nu, j}\left(e^{s}\right) d s \\
& v_{\nu, j}^{(2)}\left(e^{t}\right)=-\frac{1}{2 \nu} \int_{t}^{\infty} e^{\nu(t-s)} e^{\frac{n+2}{2} s}(V u)_{\nu, j}\left(e^{s}\right) d s
\end{aligned}
$$

$v_{\nu, j}^{(0)}$ gives the desired leading term. $v_{\nu, j}^{(1)}\left(e^{t}\right)$ and $v_{\nu, j}^{(2)}\left(e^{t}\right)$ can be bounded by

$$
\begin{aligned}
& \frac{1}{2 \nu} \int_{t}^{\infty} e^{\nu(s-t)} e^{\frac{n+2}{2} s}\left|(V u)_{\nu, j}\left(e^{s}\right)\right| d s \\
& \leq \frac{1}{2 \nu}\left\{\int_{t}^{\infty} e^{2 \nu(s-t)-2 \nu^{\prime} s} d s\right\}^{1 / 2}\left\|e^{\left(\nu^{\prime}+\frac{n+2}{2}\right) s}(V u)_{\nu, j}\right\|_{L^{2}(\mathbb{R} ; d s)} \\
& \leq C e^{-\nu^{\prime} t}\left\|r^{\nu^{\prime}+1}(V u)_{\nu, j}\right\|_{L^{2}\left(\mathbb{R}+; r^{n-1} d r\right)}
\end{aligned}
$$

with $1<\nu^{\prime}<\rho_{0}-2$. $v_{\nu, j}^{(1)}$ and $v_{\nu, j}^{(2)}$ give rise to the $L^{2}$-remainder term of $u_{\nu, j}$. (4.12) is proved, hence Theorem 4.1.

$u \in \mathcal{N} / \operatorname{ker}_{L^{2}} P$ will be called a $\nu$-resonant state of $P$ if

$$
u(r \theta)=\frac{\phi(\theta)}{r^{\frac{n-2}{2}+\nu}}+O\left(\frac{1}{r^{\frac{n-2}{2}+\nu+\epsilon}}\right), \quad r \rightarrow \infty, \quad \epsilon>0,
$$

for some $\phi \neq 0$. A family of $\nu$-resonant states is said linearly independent if their leading parts are linearly independent. A consequence of Theorem 4.1 is the following

Corollary 4.2 Let $m_{\nu} \geq 0$ denote the maximal number of linearly independent $\nu$-resonant states. Then,

$$
m_{\nu} \leq n_{\nu}
$$

where $n_{\nu}$ is the multiplicity of the eigenvalue $\lambda_{\nu}=\nu^{2}-\frac{(n-2)^{2}}{4}$ of $-\Delta_{h}+q(\theta)$. In particular, 0 is not a resonance of $P$ if $\sigma_{1}=\emptyset$.

Remark 4.3 Assume that $u$ is a $\nu$-resonant state. Assume $\rho_{0}>2$. Since $V u \in$ $H^{-1, \rho_{0}-1+\nu-\epsilon}$ for any $\epsilon>0, \nu^{\prime}$ at the end of the proof of Theorem 4.1 can be 
any number with $\nu<\nu^{\prime}<\rho_{0}-2+\nu$. This shows for $\lambda_{\nu}=\nu^{2}-(n-2)^{2} / 4$ and $u_{\nu, j}=\left(u, \varphi_{\nu}^{(j)}\right)$,

$$
u_{\nu, j}(r)=-\frac{1}{2 \nu}<V u, \varphi_{\nu}^{(j)}|y|^{-\frac{n-2}{2}+\nu}>r^{-\frac{n-2}{2}-\nu}\left(1+O\left(r^{-\epsilon}\right)\right), \quad r \rightarrow \infty,
$$

for any $0<\epsilon<\rho_{0}-2$.

Assume now $0 \in \sigma_{\infty}$. By Theorem 2.3, $R_{0}(z)=\ln z G_{0,0} \pi_{0}+F_{0}+O\left(|z|^{\epsilon}\right)$ in $\mathcal{L}(-1, s ; 1,-s)$ for any $s>1$. Let $\phi_{0}(x)=\varphi_{0}^{(1)}(\theta) r^{-\frac{n-2}{2}}$, where $\varphi_{0}^{(1)}$ is a normalized eigenfunction of $-\Delta_{h}+q(\theta)$ with eigenvalue $-(n-2)^{2} / 4$. Denote still by $\phi_{0}$ its extension by 0 outside $M_{0}$. Then

$$
G_{0,0} \pi_{0}=\frac{1}{2}<\cdot, \phi_{0}>\phi_{0}
$$

is defined on $M$.

Theorem 4.4 Assume $\rho_{0}>3$ and $0 \in \sigma_{1}$. Let $u \in \mathcal{N}$.

(a). One has

$$
\begin{aligned}
u= & <V u,-\ln r \phi_{0}>\phi_{0} \\
& +\sum_{0<\nu \leq 1} \sum_{j=1}^{n_{\nu}} \frac{1}{2 \nu}<V u,-|y|^{-\frac{n-2}{2}+\nu} \varphi_{\nu}^{(j)}>\frac{\varphi_{\nu}^{(j)}(\theta)}{r^{\frac{n-2}{2}+\nu}}+v
\end{aligned}
$$

where $v \in L^{2}$.

(b). Assume $M=M_{0}$. Let $u \in \mathcal{N}$. Then, one has $<V u, \phi_{0}>=0$ and $\left(u+F_{0} V u\right)=\beta \phi_{0}$ with $\beta=\frac{1}{2}<V u,-\ln r \phi_{0}>$.

Proof. (a). We keep the notation of Theorem 4.1. By the proof of Theorem 4.1 , it remains to study the asymptotics of $u_{0}(r)=\left(u, \varphi_{0}\right)_{\boldsymbol{\Sigma}}, u \in \mathcal{N}$, which satisfies $P_{0} u_{0}=-(V u)_{0}$. The argument used in the proof of Theorem 4.1 allows to show that there exists a constant $c$ such that $u_{0}=c r^{-\frac{n-2}{2}}+u_{0}^{\prime}$ with $u_{0}^{\prime}, \partial_{r} u_{0}^{\prime}=$ $O\left(r^{-\frac{n-2}{2}-1-\epsilon}\right)$, for some $\epsilon>0$ as $r \rightarrow \infty$. To determine $c$, we calculate for $R>1$

$$
\begin{aligned}
& \int_{r \leq R}(V u)(x) \ln r \phi_{0}(x) d x \\
& \quad=-\int_{r \leq R}\left(P_{0} u\right)_{0}(r) r^{\frac{n}{2}} \ln r d r \\
& \quad=\left.\left(\partial_{r}\left(u_{0}\right)\left(\ln r r^{n / 2}\right)-u_{0} \partial_{r}\left(\ln r r^{n / 2}\right)+(n-1) u_{0}\left(\ln r r^{n / 2-1}\right)\right)\right|_{r=R} \\
& =-c+O\left(R^{-\epsilon}\right) .
\end{aligned}
$$


Here $x=r \theta \in M_{0}$ and $d x=r^{n-1} d r d v_{h}$. Taking the limit $R \rightarrow \infty$, we obtain $c=<V u,-\ln r \phi_{0}>$.

(b). Assume $M=M_{0}$. For $u \in \mathcal{N}$, one has $\left(P_{0}+V\right) u=0$. Since $u \in H_{0}^{1,-s}$ and $P_{0} u=-V u \in H^{1, s}$ for some $s>1$, one has: $<P_{0} u, \phi_{0}>=<u, P_{0} \phi_{0}>=0$. It follows that $<V u, \phi_{0}>=0$. Let $\phi_{0}^{*} \in H^{-1, s}$ with $<\phi_{0}, \phi_{0}^{*}>=1$. Set $\pi=<\cdot, \phi_{0}^{*}>\phi_{0}, \pi^{\prime}=1-\pi$ and $u^{\prime}=\pi^{\prime} u$. Then $\pi^{\prime} G_{0,0}=G_{0,0} \pi^{\prime}=0$ and

$$
P_{0} u^{\prime}+\pi^{\prime}(V u)=0
$$

From Theorem 2.3, it follows that $u^{\prime}+F_{0} \pi^{\prime}(V u)=0$, or $\left(1+F_{0} V\right) u=\beta \phi_{0}$ for

some $\beta \in \mathbb{C}$. By (a), $u_{0}=c r^{-\frac{n-2}{2}}+u_{0}^{\prime}$ with $c$ as in (a) and $u_{0}^{\prime} \in L^{2}$. Making use of the relations $u_{0}+F_{0,0}(V u)_{0}=\beta r^{-\frac{n-2}{2}}$ and $<V u, \phi_{0}>=0$, we obtain from the asymptotic behavior of $F_{0,0}(V u)_{0}$ as $r \rightarrow \infty$ that $\frac{c}{2}=\beta$.

Part (b) of Theorem 4.4 shows that $\left(1+F_{0} V\right) \phi=0$ if $\phi \in \mathcal{N}$ is not a 0 -resonant state. If $\left(1+F_{0} V\right) u=\beta \phi_{0}$ and if

$$
\gamma_{0} \equiv<\phi_{0},-V \phi_{0}>\neq 0
$$

then, $\beta=-\gamma_{0}^{-1}<F_{0} V u, V \phi_{0}>$.

\section{A semi-classical estimate on the multiplicity of the threshold resonance}

To show that the multiplicity of the threshold resonance in geometric scattering can be arbitrarily large, we give an example in terms of Hodge-de Rham Laplacian with a metric depending on a semi-classical parameter $\hbar$. This example is based on Proposition 5.3 of [11] which is proved by Theorem of extended index $([9,17])$. Let us recall the framework in which Proposition 5.3 of [11] can be applied.

Let $(M, g)$ be a Riemannian manifold of even dimension $n$, with $M=$ $\mathbb{R}_{+} \times \Sigma, \Sigma$ being a compact $(n-1)$-dimensional Riemannian manifold without boundary. Suppose that there exists an $\hbar$-independent compact outside which 
$g=g_{0}=d r^{2}+f(r)^{2} h$, where

$$
f(r)=\hbar^{-1} r, \quad \hbar>0
$$

Let $\Lambda^{p} T^{*} M$ denote differential $p$-forms over $M$ and let

$$
\Lambda T^{*} M=\oplus_{p=0}^{n} \Lambda^{p} T^{*} M, \quad \Lambda^{e v e n} T^{*} M=\oplus_{p=0}^{\frac{n}{2}} \Lambda^{2 p} T^{*} M .
$$

Every differential form $\alpha \in \Lambda^{2 p} T^{*}\left(\mathbb{R}_{+} \times \boldsymbol{\Sigma}\right)$ can be decomposed as $\omega=d r \wedge$ $\alpha_{1}+\alpha_{2}$ with $\alpha_{1} \in \Lambda^{2 p-1} T^{*} \boldsymbol{\Sigma}$ and $\alpha_{2} \in \Lambda^{2 p} T^{*} \boldsymbol{\Sigma}$. Define

$$
I: \quad C_{0}^{\infty}\left(\Lambda T^{*}\left(\mathbb{R}_{+} \times \boldsymbol{\Sigma}\right)\right) \rightarrow C_{0}^{\infty}\left(\mathbb{R}_{+} ; \Lambda T^{*} \boldsymbol{\Sigma}\right)
$$

by $I(\alpha)=f^{\wp-(n-1) / 2} \alpha_{1}-f^{\wp-(n-1) / 2} \alpha_{2}$, where $\wp=p$ on $C_{0}^{\infty}\left(\Lambda^{p} T^{*} \boldsymbol{\Sigma}\right) . I$ extends to an isometry from $L^{2}\left(\Lambda^{\text {even }} T^{*}\left(\mathbb{R}_{+} \times \boldsymbol{\Sigma}\right)\right)$ onto $L^{2}\left(\mathbb{R}_{+} ; \Lambda T^{*} \boldsymbol{\Sigma}\right)$ :

$$
\|\omega\|_{L^{2}\left(\left(\Lambda^{\text {even }} T^{*}\left(\mathbb{R}_{+} \times \boldsymbol{\Sigma}\right)\right)\right)}^{2}=\int_{0}^{\infty}\|I(\omega)(r)\|_{L^{2}\left(\mathbb{R}_{+} ; \Lambda T^{*} \boldsymbol{\Sigma}\right)}^{2} d r .
$$

Let $D=d+\delta: \Lambda^{\text {even }} T^{*} M \rightarrow \Lambda^{\text {odd }} T^{*} M$ denote the Gauss-Bonnet operator on $M$. Then, $D$ is isometric to a Dirac-type operator outside a compact $([6])$ :

$$
I(d+\delta) I^{-1} \alpha=\sigma\left(\frac{\partial}{\partial r}+\frac{\hbar A+L}{r}\right) \alpha, \quad \alpha \in C_{0}^{\infty}\left(\mathbb{R}_{+} ; \Lambda T^{*} \boldsymbol{\Sigma}\right),
$$

where $A$ is Gauss-Bonnet operator over $\Sigma$, therefore a symmetric first order elliptic differential operator, $\sigma=(-1)^{\wp}$, and $L=(-1)^{\wp}(\wp-(n-1) / 2)$. For each fixed $\hbar, D$ is non-parabolic at infinity on $(M, g)$ (Proposition 5.5, [11]). The theorem of extended index can be applied (see $[9,17])$. Let $E_{+}, E_{-}$denote differential forms with even and odd degrees, respectively. Let $D_{ \pm}$be the restriction of $D$ to $E_{ \pm}$. In the decomposition $\Lambda T^{*} M=E_{+} \oplus E_{-}$, the Hodge-de Rham Laplacian $P=D^{2}=\delta d+d \delta$ has a supersymmetric structure. Let $P_{ \pm}$be the restriction of $P$ to $E_{ \pm}$. Then,

$$
P=\left(\begin{array}{cc}
P_{+} & 0 \\
0 & P_{-}
\end{array}\right) .
$$

Let $m_{\nu}^{ \pm}(\hbar)$ denote the dimension of $\nu$-resonant states of $P_{ \pm}$. The dimension of $\nu$-resonant states of $P$ is $m_{\nu}(\hbar)=m_{\nu}^{+}(\hbar)+m_{\nu}^{-}(\hbar)$. According to Proposition 5.3 of $[11]$

$$
m_{\nu}^{+}(\hbar)+m_{1-\nu}^{-}(\hbar)=\operatorname{dim} \operatorname{ker}\left(A(\hbar)-\left(\frac{n-2}{2}+\nu\right)\right), \quad 0 \leq \nu \leq 1,
$$


where $A(\hbar)=\hbar A+L$ can be explicitly calculated by using the Hodge operator $*_{h}$ on $\boldsymbol{\Sigma}$. See (5.10) in [6]. It follows that

$$
\sum_{0 \leq \nu \leq 1}\left(m_{\nu}^{+}(\hbar)+m_{1-\nu}^{-}(\hbar)\right)=\operatorname{dim} \chi_{[0,1]}\left(A(\hbar)-\frac{n-2}{2}\right),
$$

where $\chi_{[0,1]}$ is the characteristic function for the interval $[0,1]$.

Proposition 5.1 There exists $C_{n}>0$ such that

$$
m(\hbar) \geq C_{n} \hbar^{-(n-1)}(1+o(1)), \quad \hbar \rightarrow 0 .
$$

Proof. Note that by the asymptotic behavior of resonant states, one sees that $m_{1-\nu}^{-}(\hbar) \neq 0$ only if $\mu=1-\nu$ is an eigenvalue of $A(\hbar)$. Therefore

$$
\sum_{0 \leq \nu \leq 1} m_{1-\nu}^{-}(\hbar) \leq \sum_{0 \leq \mu \leq 1} m_{\mu}^{-}(\hbar)
$$

The total multiplicity of zero resonance of $P$ has then a lower bound

$$
m(\hbar)=\sum_{\nu}\left(m_{\nu}^{+}(\hbar)+m_{\nu}^{-}(\hbar)\right) \geq \operatorname{dim} \chi_{[0,1]}\left(A(\hbar)^{+}-\frac{n-2}{2}\right),
$$

where $\chi_{[0,1]}$ is the characteristic function for the interval $[0,1]$. It suffices to apply the result on semi-classical asymptotics of eigenvalues to calculate rank $\chi_{[0,1]}\left(A(\hbar)-\frac{n-2}{2}\right)$. In an orthonormal frame of $T_{x}^{*} \Sigma, x \in \Sigma$, the $\hbar$-principal symbol of $A(\hbar)$ is given by

$$
\left(\begin{array}{cccccc}
l_{0} & \xi \cdot & 0 & \cdots & 0 & 0 \\
\xi \wedge & l_{1} & \xi \cdot & \ddots & & 0 \\
0 & \xi \wedge & l_{2} & \ddots & \ddots & \vdots \\
\vdots & \ddots & \ddots & \ddots & \ddots & 0 \\
0 & & \ddots & \ddots & l_{n-2} & \xi \cdot \\
0 & 0 & \cdots & 0 & \xi \wedge & l_{n-1}
\end{array}\right) \quad \text { on } \quad \oplus_{p=0}^{n-1} \Lambda^{p} T_{x}^{*} \Sigma
$$

where $l_{p}=(-1)^{p}\left(p-\frac{n-1}{2}\right), \xi \wedge$ is the wedge product and $\xi \cdot$ the contraction. From the semi-classical asymptotics of eigenvalues on compact manifolds without boundary (see [13] with $\lambda=\hbar^{-1} \rightarrow \infty$ ), it follows that $m(\hbar)$ admits a lower bound of the form (5.4) with

$$
C_{n}=\int_{T^{*} \boldsymbol{\Sigma}} \operatorname{tr} \chi_{[0,1]}\left(\sigma(x, \xi)-\frac{n-2}{2}\right)>0 .
$$


Here tr denotes the trace of operators on $\oplus_{p=0}^{n-1} \Lambda^{p} T_{x}^{*} \Sigma$.

For $M=\mathbb{R}^{2}$ and $\hbar=1, \mathrm{G}$. Carron gave in $\S 5.4$ of [11] a formula for some $m_{\nu}^{-}$. Proposition 6.1 may be regarded as a more general version of this formula.

\section{A Some oscillatory integrals}

In this Appendix, we give some formulae needed to establish asymptotic expansions for the free resolvent and wave functions.

Lemma A.1 Let $\zeta \in \mathbb{C}$ with $\Im \zeta \geq 0$. Assume that $f$ is a smooth function in $s \in[0,1]$ and has the convergent expansion $f(s)=\sum_{j=0}^{\infty} s^{j} f_{j}, s \in[0,1]$. Let

$$
I_{\nu}(\zeta)=\int_{1}^{\infty} e^{i \zeta t} t^{-1-\nu} f\left(\frac{1}{t}\right) d t, \quad \nu \geq 0
$$

(a). Let $0<\nu<1$. Then,

$$
I_{\nu}(\zeta)=\sum_{j=0}^{N} \zeta^{j} C_{\nu, j}+\zeta^{\nu} \sum_{j=0}^{N} \zeta^{j} b_{\nu, j} f_{j}+\tilde{R}_{\nu, N, 2}(\zeta)
$$

Here

$$
\begin{aligned}
C_{\nu, j}= & \frac{i^{j}}{j !} \int_{1}^{\infty} t^{j-\nu-1} \mathcal{O}_{j}\left(f\left(\frac{1}{t}\right)\right) d t+\frac{i^{j}}{j !} \sum_{k=0}^{j} \frac{f_{k}}{k-j+\nu} \\
b_{\nu, j}= & -\frac{i^{j} e^{-i \nu \pi / 2} \Gamma(1-\nu)}{\nu(\nu+1) \cdots(\nu+j)}, \quad j \geq 0 \\
\tilde{R}_{\nu, N, 2}(\zeta)= & \int_{1}^{\infty} \mathcal{O}_{N}\left(e^{i \zeta t}\right) t^{-1-\nu} \mathcal{O}_{N}\left(f\left(\frac{1}{t}\right) d t\right. \\
& -\sum_{j=0}^{N-1} f_{j} \frac{(i \zeta)^{j+1}}{j !} \int_{0}^{1} \int_{0}^{1} \mathcal{O}_{N-j-1}\left(e^{i \zeta \theta t}\right)(1-\theta)^{j} t^{-\nu} d t d \theta
\end{aligned}
$$

(b). When $\nu=0$,

$$
I_{0}(\zeta)=-\ln \zeta \sum_{j=0}^{N} \zeta^{j} \frac{i^{j} f_{j}}{j !}+\sum_{j=0}^{N} \zeta^{j} C_{0, j}+(i \zeta)^{N+1} f_{N+1} b_{N+1}+\tilde{R}_{0, N, 2}(\zeta) .
$$


Here

$$
\begin{aligned}
C_{0, j}= & \frac{i^{j}}{j !} \int_{1}^{\infty} t^{j-1} \mathcal{O}_{j}\left(f\left(\frac{1}{t}\right)\right) d t+i^{j} f_{j} b_{j}+\frac{i^{j}}{j !} \sum_{k=0}^{j-1} \frac{f_{k}}{k-j}, \quad j \geq 0,(A \\
b_{0}= & \int_{1}^{\infty} e^{i t} \frac{d t}{t}+\int_{0}^{1}\left(e^{i t}-1\right) \frac{d t}{t} \\
b_{j}= & -\frac{1}{(j-1) !} \int_{0}^{1}(1-\theta)^{j-1} \ln \theta d \theta+\frac{b_{0}}{j !}, \quad j \geq 1 \\
\tilde{R}_{0, N, 2}(\zeta)= & \int_{1}^{\infty} t^{-1} \mathcal{O}_{N}\left(e^{i \zeta t}\right) \mathcal{O}_{N+1}\left(f\left(\frac{1}{t}\right)\right) d t-f_{0} \int_{0}^{1} t^{-1} \mathcal{O}_{N}\left(e^{i \zeta t}\right) d t \\
& \left.-\sum_{j=1}^{N} f_{j} \frac{(i \zeta)^{j}}{(j-1) !} \int_{0}^{1} \int_{0}^{1}(1-\theta)^{j-1} \mathcal{O}_{N-j}\left(e^{i t \zeta \theta}\right) \frac{d t}{t}\right) d \theta
\end{aligned}
$$

Proof. (a). Let $0<\nu<1$. For $\zeta \in \mathbb{C}$ with $\Im \zeta \geq 0$ and $\zeta \neq 0$, one has

$$
\int_{1}^{\infty} e^{i \zeta t} t^{-\nu} d t=\zeta^{\nu-1} \int_{0}^{\infty} e^{i t} t^{-\nu} d t-\int_{0}^{1} e^{i \zeta t} t^{-\nu} d t
$$

For $j \geq 0$, we first use the Taylor expansion with integral remainder (2.11) of order $j$ to $e^{i \zeta t}$, then use (A.11) and finally again apply the Taylor expansion of the order $N-j-1$ to $e^{i \zeta \theta t}$. By an elementary calculation, it follows that

$$
\begin{aligned}
& \int_{1}^{\infty} e^{i \zeta t} t^{-1-\nu-j} d t \\
& =\sum_{k=0}^{N} \frac{(i \zeta)^{k}}{k !(j-k+\nu)}+\zeta^{j+\nu} b_{\nu, j}-\frac{(i \zeta)^{j+1}}{j !} \int_{0}^{1} \int_{0}^{1} \mathcal{O}_{N-j-1}\left(e^{i \zeta \theta t}\right)(1-\theta)^{j} t^{-\nu} d t d \theta .
\end{aligned}
$$

Here

$$
b_{\nu, j}=\frac{i^{j+1}}{j !} \int_{0}^{1}(1-\theta)^{j} \theta^{\nu-1} d \theta \int_{0}^{\infty} e^{i t} t^{-\nu} d t .
$$

Expanding first $f\left(\frac{1}{t}\right)$ up to the order $O\left(t^{-N-1}\right)$ and then applying the above 
formula, we obtain

$$
\begin{aligned}
I_{\nu}(\zeta)= & \sum_{j=0}^{N} f_{j} \int_{1}^{\infty} e^{i \zeta t} t^{-j-1-\nu} d t+\int_{1}^{\infty} e^{i \zeta t} t^{-1-\nu} \mathcal{O}_{N}\left(f\left(\frac{1}{t}\right)\right) d t \\
= & \sum_{j=0}^{N} f_{j}\left\{\sum_{k=0}^{N} \frac{(i \zeta)^{k}}{k !(j-k+\nu)}+\zeta^{j+\nu} b_{\nu, j}\right\}+\sum_{j=0}^{N}(i \zeta)^{j} C_{j, \nu, N} \\
& +\int_{1}^{\infty} \mathcal{O}_{N}\left(e^{i \zeta t}\right) t^{-1-\nu} \mathcal{O}_{N}\left(f\left(\frac{1}{t}\right) d t\right. \\
& -\sum_{j=0}^{N} f_{j} \frac{(i \zeta)^{j+1}}{j !} \int_{0}^{1} \int_{0}^{1} \mathcal{O}_{N-j-1}\left(e^{i \zeta \theta t}\right)(1-\theta)^{j} t^{-\nu} d t d \theta \quad \text { with } \\
C_{j, \nu, N}= & \frac{1}{j !} \int_{1}^{\infty} t^{j-\nu-1} \mathcal{O}_{N}\left(f\left(\frac{1}{t}\right)\right) d t .
\end{aligned}
$$

It follows that

$$
I_{\nu}(\zeta)=\sum_{j=0}^{N}(i \zeta)^{j}\left(C_{j, \nu, N}+\frac{1}{j !}\left(\sum_{k=0}^{N} \frac{f_{k}}{k-j+\nu}\right)\right)+\zeta^{\nu} \sum_{j=0}^{N} \zeta^{j} b_{\nu, j} f_{j}+\tilde{R}_{\nu, N, 2}(\zeta) .
$$

(a) follows by noticing that to obtain the formula for $C_{\nu, j}$, we need only to apply (A.12) with $N=j$.

To prove (b), we use the identity

$$
\int_{1}^{\infty} e^{i t \zeta} \frac{d t}{t}=-\ln \zeta+b_{0}+\int_{0}^{1}\left(1-e^{i t \zeta}\right) \frac{d t}{t}
$$

with $b_{0}$ defined in Lemma A.1. It follows that

$$
\begin{aligned}
I_{0}(\zeta) & =\sum_{j=0}^{N+1} f_{j} \int_{1}^{\infty} t^{-j-1} e^{i \zeta t} d t+\int_{1}^{\infty} t^{-1} e^{i \zeta t} \mathcal{O}_{N+1}\left(f\left(\frac{1}{t}\right)\right) d t \\
& =\sum_{j=0}^{N+1} f_{j} \int_{1}^{\infty} t^{-j-1} e^{i \zeta t} d t+\sum_{j=0}^{N}(i \zeta)^{j} C_{j, 0, N}+\int_{1}^{\infty} t^{-1} \mathcal{O}_{N}\left(e^{i \zeta t}\right) \mathcal{O}_{N+1}\left(f\left(\frac{1}{t}\right)\right) d t \\
C_{j, 0, N} & =\frac{1}{j !} \int_{1}^{\infty} t^{j-1} \mathcal{O}_{N+1}\left(f\left(\frac{1}{t}\right)\right) d t
\end{aligned}
$$


For $j \geq 1$, one has

$$
\begin{aligned}
\int_{1}^{\infty} & e^{i t \zeta} \frac{d t}{t^{j+1}} \\
= & \sum_{k=0}^{j-1} \frac{(i \zeta)^{k}}{k !(j-k)}+\frac{(i \zeta)^{j}}{(j-1) !} \int_{0}^{1}(1-\theta)^{j-1}\left(\int_{1}^{\infty} e^{i t \theta \zeta} \frac{d t}{t}\right) d \theta \\
= & \sum_{0 \leq k \leq N, k \neq j} \frac{(i \zeta)^{k}}{k !(j-k)}+(i \zeta)^{j}\left(-\frac{\ln \zeta}{j !}+b_{j}\right) \\
& \left.-\frac{(i \zeta)^{j}}{(j-1) !} \int_{0}^{1} \int_{0}^{1}(1-\theta)^{j-1} \mathcal{O}_{N-j}\left(e^{i t \zeta \theta}\right) \frac{d t}{t}\right) d \theta
\end{aligned}
$$

where

This proves

$$
b_{j}=-\frac{1}{(j-1) !} \int_{0}^{1}(1-\theta)^{j-1} \ln \theta d \theta+\frac{b_{0}}{j !} .
$$

$$
\begin{aligned}
I_{0}(\zeta)= & f_{0}\left(-\ln \zeta+b_{0}-\sum_{k=1}^{N} \frac{(i \zeta)^{k}}{k ! k}\right)+\sum_{j=0}^{N}(i \zeta)^{j} C_{j, 0, N}+\sum_{j=1}^{N+1}(i \zeta)^{j} f_{j}\left(b_{j}-\frac{\ln \zeta}{j !}\right) \\
& +\sum_{0 \leq k \leq N}(i \zeta)^{k} \sum_{1 \leq j \leq N+1, j \neq k} \frac{f_{j}}{k !(j-k)}+\tilde{R}_{0, N, 2}(\zeta)
\end{aligned}
$$

with $\tilde{R}_{0, N, 2}$ given in Lemma A.1. The expression for $C_{0, j}$ follows by applying the above formula with $N=j$.

To obtain long-time expansions, we need the following results on the Fourier transform of temperate distributions of the form $(s+i 0)^{\lambda} \ln ^{k}(s+i 0)$.

Lemma A.2 (a). For $\lambda \in \mathbb{R}, k \in \mathbb{N}$,

$$
\begin{aligned}
& \frac{1}{2 \pi i} \int(s+i 0)^{\lambda} \ln ^{k}(s+i 0) e^{-i t s} d s \\
& \quad=\frac{i}{\pi} \sum_{j=0}^{k} C_{k}^{j}\left(\frac{d^{k-j}}{d \lambda^{k-j}}\left(\sin (\pi \lambda) e^{i \pi \lambda / 2} \Gamma(\lambda+1)\right)\right) t^{-1-\lambda} \ln ^{j} t
\end{aligned}
$$

for $t>0$.

(b). For $\lambda \in \mathbb{R}, k \in \mathbb{N}^{*}$,

$$
\begin{aligned}
& \frac{1}{2 \pi i} \int(s+i 0)^{\lambda} \ln ^{-k}(s+i 0) e^{-i t s} d s \\
& =t^{-\lambda-1} \frac{i(-1)^{k}}{\pi}\left\{\sum_{l=k}^{N} C_{l+k-1}^{k-1} \frac{d^{l-k}}{d \lambda^{l-k}}\left(\sin (\pi \lambda) e^{i \pi \lambda / 2} \Gamma(\lambda+1)\right) \ln ^{-l} t+O\left(\ln ^{-N-1} t\right)\right\}
\end{aligned}
$$


for $t>0$.

(c). Assume that $r(z)$ is an analytic function for $z$ near 0 with $\Im z>0$ and is continuous up to $\Im z=0$ satisfying $|r(z)| \leq C|z|^{\lambda}|\ln z|^{k}$. Let $\chi$ be a smooth function on $\mathbb{R}$ with support near 0 . Then

$$
\left|\frac{1}{2 \pi i} \int w(s+i 0) e^{-i t s} \chi(s) d s\right| \leq C t^{-\lambda-1} \ln ^{k} t
$$

for $t>>1$.

See [12] for (a), [20] for (b) and Lemma 10 in [22] for (c). For the long-time expansions of wave functions, we actually use the asymptotic expansions for the Fourier transform of $\chi(s)(s+i 0)^{\lambda} \ln ^{k}(s+i 0)$, where $\chi \in C_{0}^{\infty}(\mathbb{R})$ with $\chi(s)=1$ near 0. It is clear that the asymptotic expansions are the same as in (a) and (b) of Lemma A.2 for $k \geq 0$ and $k<0$.

\section{References}

[1] Albeverio, S.; Bollé, D.; Gesztesy, F.; Hoegh-Krohn, R., Low-energy parameters in nonrelativistic scattering theory, Ann. Physics, 148 (1983), 308-326.

[2] Amado, R. D.; Greenwood, F. C., There is no Efimov effect for four or more particles, Phys. Rev. D, 7(3) (1973), 2517-2519.

[3] Atiyah, M. F.; Patodi, V. K.; Singer, I. M., Spectral asymmetry and Riemannian geometry I, Math. Proc. Cambridge Phil. Soc., 77 (1975), 43-69.

[4] Bollé, D.; Gesztesy, F.; Danneels, C., Threshold scattering in two dimensions, Ann. Inst. H. Poincaré, Sect. A, 48 (1988), 175-204.

[5] Bolley, P.; Camus, J., Opérateurs à indices, Publication des Séminaires Mathématiques, Fascule II. -Séminaire d'Analyse Fonctionnelle, Univ. Rennes 1, 1973.

[6] Brüning, J.; Seeley, R. T., An index theorem for first order regular singular operators, Amer. J. Math., 110 (1988), 659-714. 
[7] Burq, N.; Planchon, F.; Stalke, J. G.; Shadi Tahvildar-Zadeh, A., Strichartz estimates for the wave and Schrödinger equations with the inverse-square potentials, J. of Funct. Analysis, 203 (2003), 519-549.

[8] Cardoso, F.; Vodev, G., Uniform estimates of the resolvent of the LaplaceBeltrami operator on infinite volume Riemannian manifolds. II, Annales Henri Poincaré, 3 (2002), 673-691.

[9] Carron, G., Théorème de l'indice sur les variétés non-compactes, J. Reine und Angew. Math., 541 (2001), 81-115.

[10] Carron, G., A topological criterion for the existence of half-bound states, J. of the London Math. Soc., 65 (2002), 757-768.

[11] Carron, G., Le saut en zéro de la fonction de décalage spectral, J. of Funct. Analysis., 212 (2004), 222-260.

[12] Gel'fand, I. M.; Shilov, G. E., Generalized Functions I, Academic Press, New York, 1964.

[13] Hörmander, L., The spectral function of an elliptic operator, Acta Math., 121 (1968), 193-218.

[14] Jensen, A.; Kato, T., Spectral properties of Schrödinger operators and time decay of wave functions, Duke Math. J., 46 (1979), 583-611.

[15] Jensen, A.; Nenciu, G., A unified approach to resolvent expansions at thresholds, Reviews in Math. Phys., 13 (2001), 717-754.

[16] Maz'ya, V.; Nazarov, S.; Plamenevskij, B., Asymptotic Theory of Elliptic Boundary Value Problems in Singularly Perturbed Domains, Volume 1. Birkhäuser, Boston-Berlin, 2000.

[17] Melrose, R. B., The Atiyah-Patodi-Singer index theorem, A. K. Peters, 1993. 
[18] Melrose, R. B., Spectral and scattering theory for the Laplacian on asymptotically Euclidean spaces, pp 85-130, in Spectral and Scattering Theory, ed. M. Ikawa, Marcel Dekker, 1994.

[19] Newton, R. G., Scattering Theory of Waves and Particles, Springer Verlag, Berlin, 1982.

[20] Olver, F. W. J., Asymptotics and Special Functions, A. K. Peters Classics, Massachusetts, 1997.

[21] Taylor, M., Partial Differential Equations II, Springer Verlag, 1996.

[22] Vainberg, B. R., On the short wave asymptotic behaviour of solutions of stationary problems and the asymptoic behaviour as $t \rightarrow \infty$ of solutions of non-stationary problems, Russ. Math. Survey, 30 (1975), 1-58.

[23] Wang, X. P., Asymptotic behavior of the resolvent of $\mathrm{N}$-body Schrödinger operators near a threshold, Ann. Henri Poincaré, 4 (2003), 553-600.

[24] Wang, X. P., On the existence of the N-body Efimov effect, J. Funct. Analysis, 209(2004), 137-161.

[25] Wang, X. P., Asymptotic expansions in time of wave functions in geometric scattering, Preprint, 2003.

[26] Waston, G. N., A Treatise on the Theory of Bessel Functions, Cambridge Univ. Press, Cambridge, 1994.

Département de Mathématiques

Université de Nantes

F-44322 Nantes Cedex 3, FRANCE

E-mail: wang@math.univ-nantes.fr 